\title{
The association between general practitioner regularity of care and 'high use' hospitalisation
}

\author{
Rachael E. Moorin ${ }^{1,2}$, David Youens ${ }^{1}$, David B. Preen ${ }^{2}$ and Cameron M. Wright ${ }^{1,3^{*}}$ (D)
}

\begin{abstract}
Background: In Australia, as in many high income countries, there has been a movement to improve out-ofhospital care. If primary care improvements can yield appropriately lower hospital use, this would improve productive efficiency. This is especially important among 'high cost users', a small group of patients accounting for disproportionately high hospitalisation costs. This study aimed to assess the association between regularity of general practitioner (GP) care and 'high use' hospitalisation.

Methods: This retrospective, cohort study used linked administrative and survey data from the 45 and Up Study, conducted in New South Wales, Australia. The exposure was regularity of GP care between 1 July 2005 and 30 June 2009 , categorised by quintile (lowest to highest). Outcomes were 'high use' of hospitalisation (defined as $\geq 3$ and $\geq$ 5 admissions within 12 months), extended length of stay (LOS, $\geq 30$ days), a combined metric ( $\geq 3$ hospitalisations in a 12 month period where $\geq 1$ hospitalisation was $\geq 30$ days) and 30-day readmission between 1 July 2009 and 31 December 2017. Associations were assessed using multivariable logistic regression. Potential for outcome prevention in a hypothetical scenario where all individuals attain the highest GP regularity was estimated via the population attributable fraction (PAF).
\end{abstract}

Results: Of 253,500 eligible participants, $15 \%$ had $\geq 3$ and $7 \%$ had $\geq 5$ hospitalisations in a 12-month period. Five percent of the cohort had a hospitalisation lasting $\geq 30$ days and $25 \%$ had a readmission within 30 days. Compared with lowest regularity, highest regularity was associated with between $6 \%(p<0.001)$ and $11 \%(p=0.027)$ lower odds of 'high use'. There was a $7-8 \%$ reduction in odds for all regularity levels above 'low' regularity for LOS $\geq 30$ days. Otherwise, there was no clear sequential reduction in 'high use' with increasing regularity. The PAF associated with a move to highest regularity ranged from 0.05 to 0.13 . The number of individuals who could have had an outcome prevented was estimated to be between 269 and 2784, depending on outcome.

Conclusions: High GP regularity is associated with a decreased likelihood of 'high use' hospitalisation, though for most outcomes there was not an apparent linear association with regularity.

Keywords: Primary health care, Chronic disease, Continuity of care, Hospitalisation, Hospital costs, health policy

\footnotetext{
* Correspondence: cameron.wright@curtin.edu.au

${ }^{1}$ Health Economics and Data Analytics, School of Public Health, Faculty of

Health Sciences, Curtin University, GPO Box U1987, Perth, Western Australia 6845, Australia

${ }^{3}$ School of Medicine, College of Health \& Medicine, University of Tasmania, Hobart, Tasmania, Australia

Full list of author information is available at the end of the article
}

(c) The Author(s). 2020 Open Access This article is licensed under a Creative Commons Attribution 4.0 International License, which permits use, sharing, adaptation, distribution and reproduction in any medium or format, as long as you give appropriate credit to the original author(s) and the source, provide a link to the Creative Commons licence, and indicate if changes were made. The images or other third party material in this article are included in the article's Creative Commons licence, unless indicated otherwise in a credit line to the material. If material is not included in the article's Creative Commons licence and your intended use is not permitted by statutory regulation or exceeds the permitted use, you will need to obtain permission directly from the copyright holder. To view a copy of this licence, visit http://creativecommons.org/licenses/by/4.0/ The Creative Commons Public Domain Dedication waiver (http://creativecommons.org/publicdomain/zero/1.0/) applies to the data made available in this article, unless otherwise stated in a credit line to the data. 


\section{Background}

In Australia, as in many high income countries, there has been a movement to improve out-of-hospital care $[1,2]$. The Australian 'Chronic Disease Management' financial incentive program aims to foster a structured approach to general practitioner (GP)-provided primary care [3]. If primary care improvements can yield appropriately lower hospital use, this would improve productive efficiency (minimal resources for maximal output) [4]. This is especially important among 'high cost users', a small group of patients accounting for disproportionately high hospitalisation costs $[5,6]$.

In order to evaluate primary health funding incentives, researchers have analysed continuity of care on a range of health services use and outcomes [7-9]. This typically refers to the proportion of care provided by a single provider (e.g. usual provider of care index (UPC) [10]) or the distribution of care among providers (e.g. the Modified Modified Continuity Index (MMCI) [11]). More recently, work from Australia has assessed GP 'regularity' as a possible factor for improving tertiary disease prevention (i.e. reducing complications requiring hospital care) [12-16]. This concept assesses how evenly distributed care provision by any GP is across time. This has relevance in Australia where a feefor-service model has encouraged reactive care and to settings such as the United Kingdom (UK) $[17,18]$ and United States (U.S) [19], where the literature documents falling continuity of provider. Work using a regularity index among a cohort with diabetes has found an association between higher GP regularity and decreased rate and cost of diabetes-related hospitalisations [16]. One of the issues in assessing regularity is in disentangling its effects from those of continuity of provider and frequency of care. Again, among a cohort of people with diabetes, higher regularity (adjusted for continuity of provider and frequency of GP attendance) showed an association with lower costs for unplanned hospitalisation, suggesting that regularity is a discrete facet of continuity of care [20]. Previous analyses have suggested that regular primary care provision may also be associated with fewer readmissions [21-24].

Given recent interest in 'high cost users' of hospitals [25], this study aimed to answer the question: Is regularity of GP-provided primary care associated with 'high use' hospitalisation?

\section{Methods}

This paper follows the REporting of studies Conducted using Observational Routinely-collected health Data (RECORD) statement [26].

\section{Data sources}

This study used individual-level self-reported data from the 45 and Up Study linked with routinely collected administrative health data [27].
The Sax Institute's 45 and Up Study is a longitudinal study of 267,153 participants, aged $\geq 45$ years in New South Wales (NSW), Australia. Prospective participants were randomly sampled from the Australian Government Department of Human Services (DHS), formerly Medicare Australia, enrolment database and recruited from January 2006 to December 2009. There was deliberate oversampling for people $\geq 80$ years and from rural areas [27]. The study methods are described in detail elsewhere [27]. Briefly, participants completed a detailed baseline health and lifestyle questionnaire. The response rate was $18 \%$ [27].

The linked data sources utilised included: (i) the 45 and Up Study baseline questionnaire (https://www.saxinstitute.org.au/our-work/45-up-study/); (ii) the NSW Admitted Patient Data Collection (APDC), which provided all hospital separations in public and private hospitals in NSW (2005-2017); (iii) the Pharmaceutical Benefits Scheme (PBS) which provided information on government-subsidised prescription medicines dispensed (2005-2017); (iv) the Medicare Benefits Schedule (MBS) which provided records for all claims for medical services (including GP consultations) provided through Medicare, Australia's universal health insurance scheme (2005-2017); and (v) the NSW Register of Births Deaths and Marriages (RBDM) (2006-2017). The linkage of APDC and RBDM to the 45 and Up Study cohort survey data was conducted by the NSW Centre for Health Record Linkage. MBS and PBS data were linked by the Sax Institute using a unique identifier provided to the Department of Human Services. Quality assurance data on the linkage show false-positive and false-negative rates of $<0.5$ and $<0.1 \%$, respectively [28].

Ethical approval was obtained from Curtin University Human Research Ethics Committee (RD-42-14) and the NSW Population and Health Services Research Ethics Committee (HREC/17/CIPHS/37). Consent was given by participants in the 45 and Up Study for their information to be used in approved studies, and for follow-up and data linkage. The conduct of the 45 and Up Study was approved by the University of NSW Human Research Ethics Committee.

\section{Participants}

All participants in the 45 and Up Study recruited prior to 1 July 2009 and still alive on 1 July 2012 were included, allowing $\geq 3$ years for outcome follow-up.

\section{Study design}

This was a retrospective, cohort study. The main exposure, GP regularity, and health service utilisation covariates were captured from 1 July 2005 to 30 June 2009. Sociodemographic covariates were obtained from the 45 and Up Study baseline questionnaire. Outcomes (i.e. 
'high use' hospitalisation) were captured from 1 July 2009 to the first of 31 December 2017 or death.

\section{GP regularity}

The number of days with GP contact, principally visiting a GP practice, was captured via MBS claims for 'Attendances by General Practitioners', which captures all GP attendances in Australia [29]. Regularity of GP contact was measured based on the number of days between each GP contact and the contact prior. For each individual, the regularity index was calculated based on this set of intervals using the previously reported Modified Regularity Index as follows (further details in Additional file 1):

$$
R_{c v}=1 /(1+c v(\text { days }))
$$

Where $c v$ is the coefficient of variation. The index therefore captures dispersion of GP contacts based on the coefficient of variation in the number of days between GP contacts within the exposure period. This score ranges from 0 to 1 , though for the analysis was divided into quintiles using the range of scores observed in the study population, treating regularity as an ordinal variable (lowest (reference) to highest). The Modified Regularity Index is a second iteration of an earlier index and is less correlated with frequency of GP contact [16]. Participants with $<3$ GP visits were categorised separately because a regularity score could not be calculated for these individuals [30, 31].

\section{Outcomes}

Binary (yes/no) outcomes for high use hospitalisation were based on previous Canadian work [25] and work by Graham and colleagues [32]. High use hospitalisation was defined as multiple hospitalisations (two outcomes: $\geq 3$ or $\geq 5$ hospitalisations over 12 months); extended length of stay (LOS, a hospitalisation with $\geq 30$ bed days) and a combined metric ( $\geq 3$ hospitalisations in a 12 month period where $\geq 1$ hospitalisation was $\geq 30$ days) [25]. The reason for multiple indicators is that these capture different subpopulations of 'high users', with the combined metric capturing a range of 'high user' conditions [25]. Readmissions within 30 days of separation (discharge) were captured overall and further categorised as either early ( $1-7$ days) or late (8-30 days) [32]. Each outcome was captured based on (i) any hospitalisation and (ii) unplanned hospitalisation, using the APDC 'emergency status' variable. Inter-hospital transfers were counted as a single hospitalisations, to avoid doublecounting.

\section{Covariates}

Self-report information on potential confounders were obtained from the 45 and Up Study baseline questionnaire. These were: age; sex; marital status; whether born in Australia; Indigenous status; current housing; household income; highest education level; smoking status; alcoholic consumption; physical activity [33]; time spent sitting; body mass index; psychological distress [34]; level of limitation and self-rated overall health and quality of life (based on 36 Item Short Form survey [35]); social support [36]; and previous diagnosis for chronic conditions (see Additional file 2 for categories). Area-based socio-economic status and residential remoteness (as a proxy for health care accessibility) were obtained from national indices [37, 38]. Comorbidity was ascertained using the Multipurpose Australian Comorbidity Scoring System (MACSS) [39], defined as the sum of comorbidities recorded in hospital records at one and 5 years prior to the start of follow-up [40]. PBS data were used to calculate the Rx-Risk comorbidity index (i.e. number of condition groups for which medicines were dispensed) at one and 5 years prior to the start of follow-up, to capture comorbidity in the out of hospital setting [41]. Continuity of provider was measured using the UPC [10] and the MMCI [11] calculated using de-identified MBS provider numbers (see Additional file 1). Use of specialist physician services, chronic disease management items and mental health-related services was captured using MBS claims data. A binary variable captured death after 1 July 2012. The number of days out of hospital during the exposure period was used to adjust for prior hospital use.

\section{Statistical analysis}

Analyses were undertaken using Stata MP Version 16.0 [42]. Descriptive statistics were stratified by regularity quintile. The associations between exposure and outcomes were assessed separately for each outcome using multivariable logistic regression, incorporating robust standard errors. To account for differential follow-up, person-time at risk of the outcome event, defined as the $\log$ of the number of days alive during follow-up, was included as an offset variable.

Finally, the potential for prevention of outcomes in a hypothetical situation (scenario 1) where all individuals attain the highest GP regularity was explored using the user-written Stata package - punaf- [43]. For each outcome the population unattributable fraction (PUF) was calculated as the ratio of the means of two competing scenarios (i.e. scenario 1 and data as observed). The PUF represented the fraction that would remain if individuals moved to scenario 1; the population attributable fraction (PAF) was 1 - PUF. 


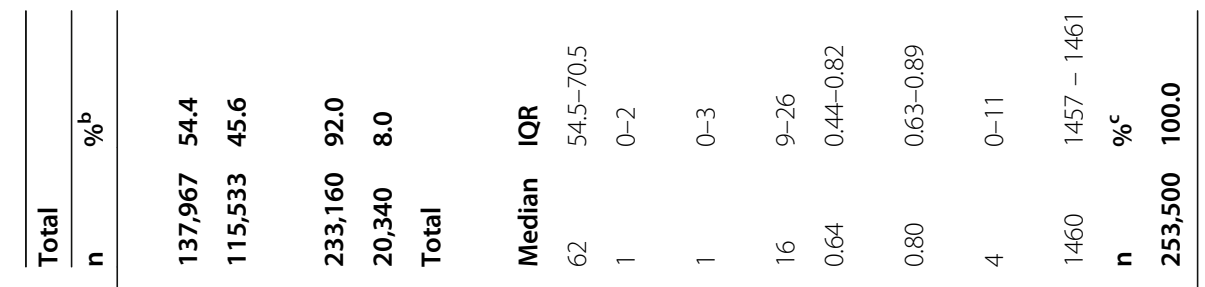

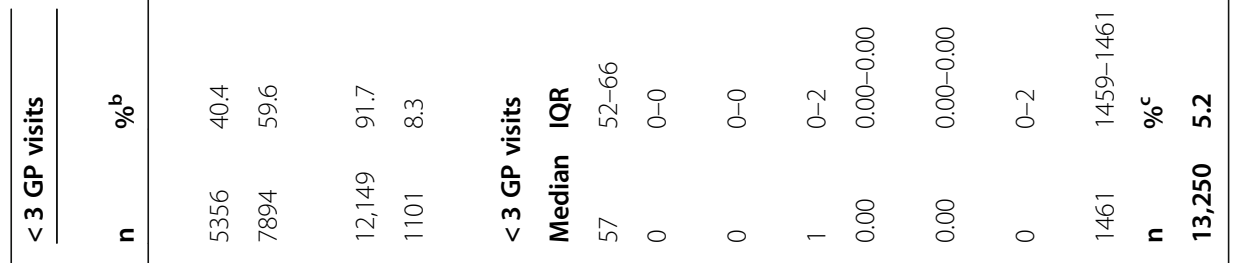

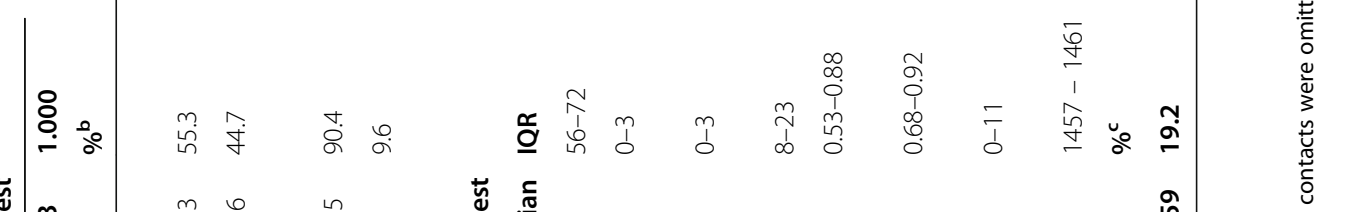

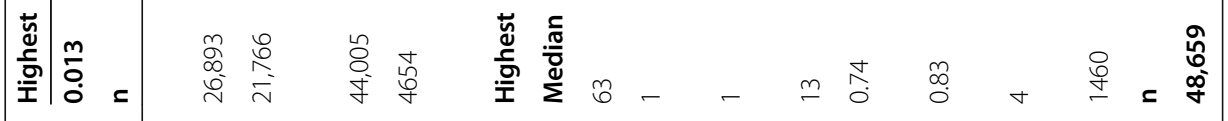

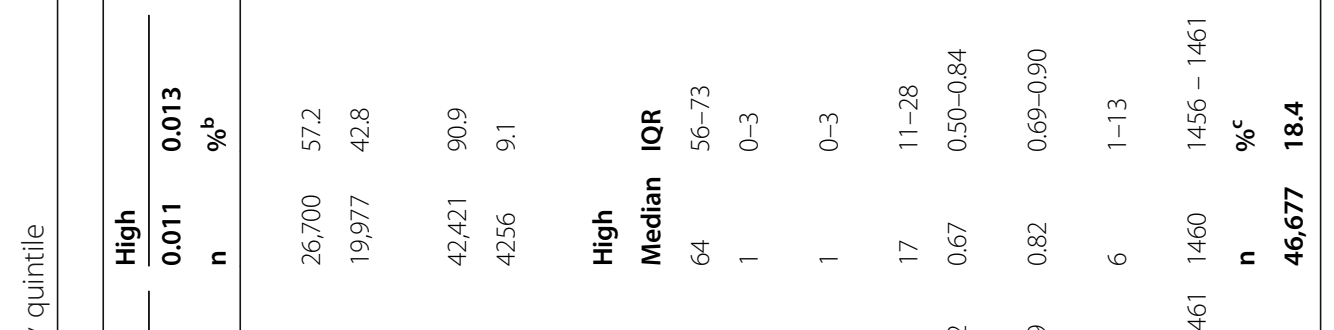

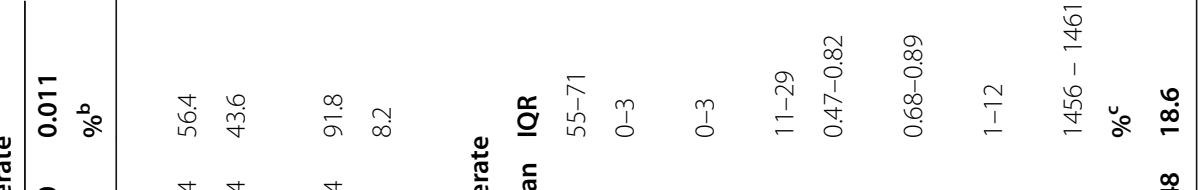

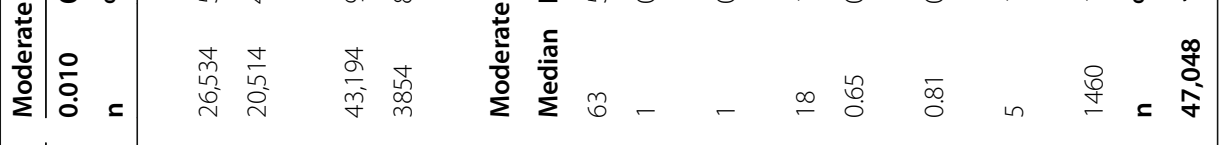

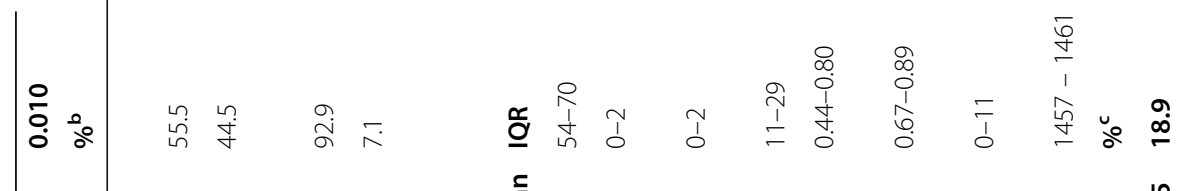

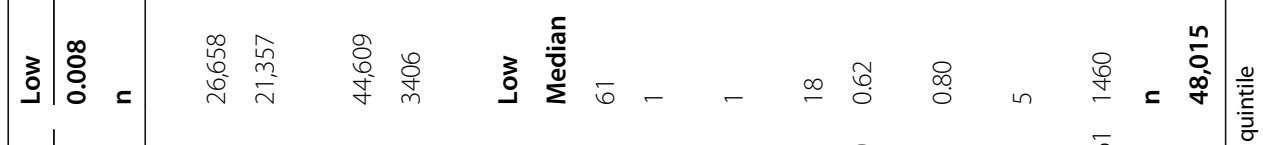

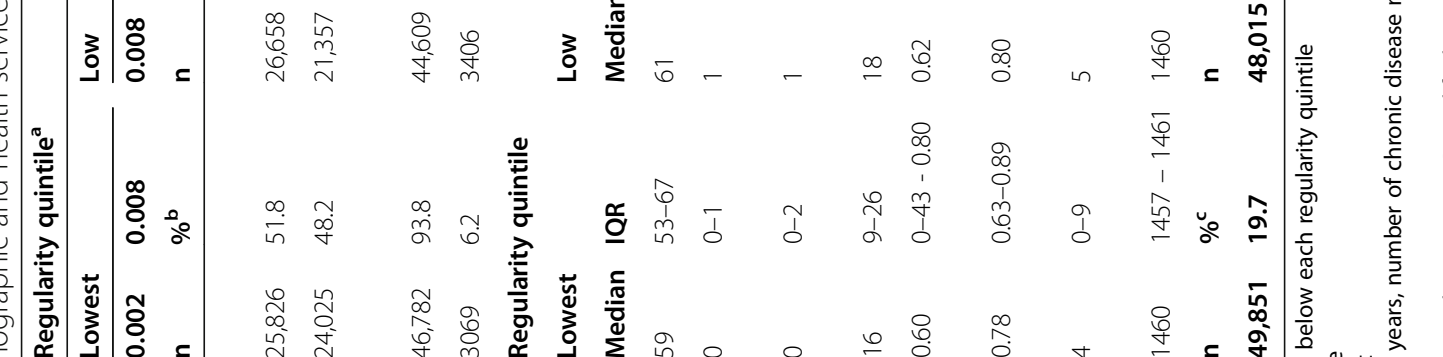

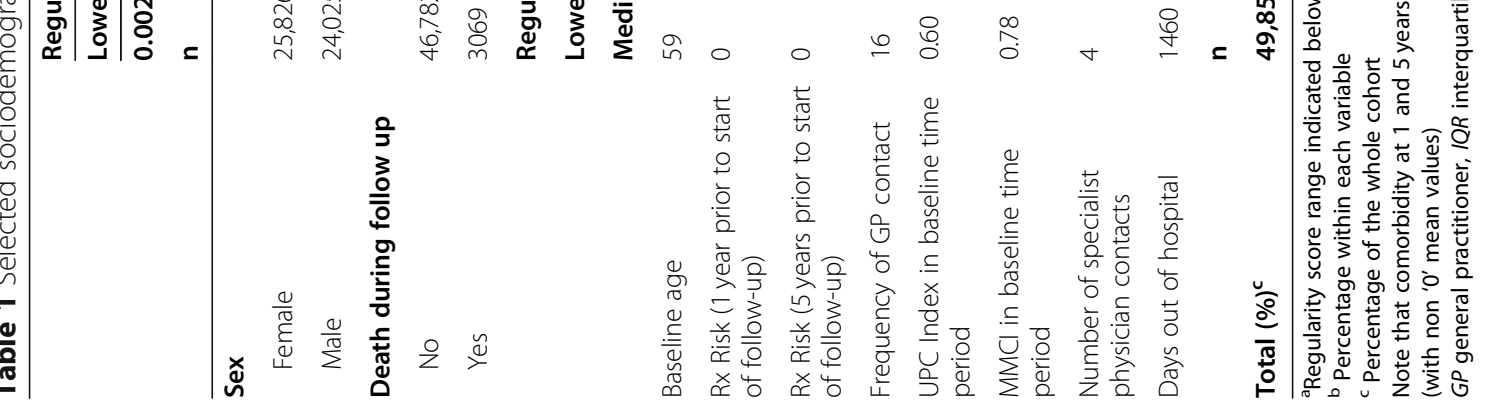




\section{Results}

Of the 254,140 participants in the 45 and Up Study recruited and still alive at 1 July 2012, 640 (0.3\%) were excluded due to a potential linkage error, leaving 253,500 eligible participants. Study participants were predominantly female (54\%); the median age at baseline was 62 years (Table 1). Seventy one percent of participants had at least one self-reported medical diagnosis (self-report and sociodemographic variables in Additional file 3). There was a relatively even distribution of socioeconomic status quintile, however, the majority (52\%) resided in highly accessible geographic areas. Participants had a median of 16 GP attendances and the median UPC was 0.64 . On average participants were in hospital for $1 \%$ of the exposure period and $8 \%$ of the cohort died during follow-up.

In total, $15 \%(n=37,931)$ of the cohort had $\geq 3$ hospitalisations in any 12 month period, of which $33 \%$ were unplanned $(n=12,857)$ (Table 2). Seven percent of the cohort had $\geq 5$ hospitalisations within 12 months $(n=17$, $767)$, of which $22 \%(n=3989)$ were unplanned. Five percent had a hospitalisation lasting $\geq 30$ days. Twenty five percent of individuals experienced a readmission within
30 days during follow up $(n=62,623)$, of which $31 \%(n=$ 19,430 ) were unplanned. Individuals experiencing an early (1-7 days) readmission accounted for $13 \%$ of the cohort, while $19 \%$ experienced a late (8-30 days) readmission. Individuals could fulfil both the early and late readmission outcome during the outcome ascertainment period.

Individuals who had the highest GP regularity had lower odds, compared with their counterparts in the lowest quintile (reference for all results), for both $\geq 3$ (adjusted odds ratio (OR) $0.94,95 \%$ confidence interval (CI) $0.90-0.98$ ) and $\geq 5$ hospitalisations (OR 0.94, 95\% CI 0.89-0.99) (Fig. 1, Additional file 4). Lower ORs were observed for unplanned hospitalisations for both outcomes: $\geq 3$ (OR 0.87, 95\% CI 0.81-0.93) and $\geq 5$ (OR 0.85, 95\% CI 0.75-0.96) hospitalisations. Conversely, moderate regularity had an OR of 1.06 (95\% CI $1.02-$ 1.10) for $\geq 3$ and 1.08 (95\% CI 1.02-1.14) for $\geq 5$ hospitalisations.

Being in the moderate (OR 0.92, 95\% CI 0.86-0.99), high (OR 0.93, 95\% CI 0.87-1.00) and highest (OR 0.92, $95 \%$ CI $0.85-0.98$ ) quintiles of regularity was associated with a lower odds of any hospitalisation with LOS $\geq 30$

Table 2 'High use' outcomes between 1 July 2009 and 31 December 2017, by regularity quintile

\begin{tabular}{|c|c|c|c|c|c|c|c|c|c|c|c|c|c|c|}
\hline & \multicolumn{12}{|c|}{ Regularity quintile } & \multirow{2}{*}{\multicolumn{2}{|c|}{ Total }} \\
\hline & \multicolumn{2}{|c|}{ Lowest } & \multicolumn{2}{|l|}{ Low } & \multicolumn{2}{|c|}{ Moderate } & \multicolumn{2}{|l|}{ High } & \multicolumn{2}{|c|}{ Highest } & \multicolumn{2}{|c|}{$\begin{array}{l}<3 \mathrm{GP} \\
\text { visits }\end{array}$} & & \\
\hline & $\mathrm{n}$ & $\%^{a}$ & $\mathrm{n}$ & $\%^{a}$ & $\mathrm{n}$ & $\%^{a}$ & $n$ & $\%^{\mathrm{a}}$ & $\mathrm{n}$ & $\%^{a}$ & $\mathrm{n}$ & $\%^{a}$ & $\mathrm{n}$ & $\%^{a}$ \\
\hline \multicolumn{15}{|l|}{ High use hospitalisation } \\
\hline$\geq 3$ hospitalisations & 6337 & 12.7 & 7118 & 14.8 & 7704 & 16.4 & 7957 & 17.0 & 7304 & 15.0 & 1511 & 11.4 & 37,931 & 15.0 \\
\hline$\geq 3$ unplanned hospitalisations & 2017 & 4.0 & 2382 & 5.0 & 2566 & 5.5 & 2732 & 5.9 & 2574 & 5.3 & 586 & 4.4 & 12,857 & 5.1 \\
\hline$\geq 5$ hospitalisations & 2960 & 5.9 & 3361 & 7.0 & 3656 & 7.8 & 3667 & 7.9 & 3360 & 6.9 & 763 & 5.8 & 17,767 & 7.0 \\
\hline$\geq 5$ unplanned hospitalisations & 627 & 1.3 & 756 & 1.6 & 769 & 1.6 & 844 & 1.8 & 788 & 1.6 & 205 & 1.5 & 3989 & 1.6 \\
\hline$\geq 30$ days length of stay & 1933 & 3.9 & 2178 & 4.5 & 2343 & 5.0 & 2622 & 5.6 & 2701 & 5.6 & 668 & 5.0 & 12,445 & 4.9 \\
\hline$\geq 30$ days length of stay (unplanned) & 1519 & 3.0 & 1762 & 3.7 & 1894 & 4.0 & 2131 & 4.6 & 2203 & 4.5 & 553 & 4.2 & 10,062 & 4.0 \\
\hline $\begin{array}{l}\geq 3 \text { hospitalisations with at least one } \\
\geq 30 \text { days }\end{array}$ & 858 & 1.7 & 943 & 2.0 & 1040 & 2.2 & 1129 & 2.4 & 1155 & 2.4 & 308 & 2.3 & 5433 & 2.1 \\
\hline $\begin{array}{l}\geq 3 \text { unplanned hospitalisations with } \\
\text { at least one } \geq 30 \text { days }\end{array}$ & 433 & 0.9 & 562 & 1.2 & 586 & 1.2 & 636 & 1.4 & 646 & 1.3 & 185 & 1.4 & 3048 & 1.2 \\
\hline \multicolumn{15}{|l|}{ Readmission } \\
\hline Early readmission (1-7 days) ${ }^{b}$ & 5811 & 11.7 & 6453 & 13.4 & 6813 & 14.5 & 6838 & 14.6 & 6407 & 13.2 & 1329 & 10.0 & 33,651 & 13.3 \\
\hline Early unplanned readmission $(1-7 \text { days })^{b}$ & 1841 & 3.7 & 2016 & 4.2 & 2133 & 4.5 & 2199 & 4.7 & 2129 & 4.4 & 450 & 3.4 & 10,768 & 4.2 \\
\hline Late readmission (8-30 days) ${ }^{b}$ & 8405 & 16.9 & 9345 & 19.5 & 9694 & 20.6 & 10,000 & 21.4 & 9373 & 19.3 & 1892 & 14.3 & 48,709 & 19.2 \\
\hline $\begin{array}{l}\text { Late unplanned readmission (8-30 } \\
\text { days) }\end{array}$ & 2135 & 4.3 & 2418 & 5.0 & 2511 & 5.3 & 2782 & 6.0 & 2552 & 5.2 & 570 & 4.3 & 12,968 & 5.1 \\
\hline Readmission within 30 days & 10,896 & 21.9 & 12,066 & 25.1 & 12,468 & 26.5 & 12,779 & 27.4 & 12,013 & 24.7 & 2401 & 18.1 & 62,623 & 24.7 \\
\hline Unplanned readmission within 30 days & 3266 & 6.6 & 3632 & 7.6 & 3803 & 8.1 & 4086 & 8.8 & 3812 & 7.8 & 831 & 6.3 & 19,430 & 7.7 \\
\hline TOTAL & $\begin{array}{l}49 \\
851\end{array}$ & 19.7 & $\begin{array}{l}48 \\
015\end{array}$ & 18.9 & $\begin{array}{l}47 \\
048\end{array}$ & 18.6 & $\begin{array}{l}46 \\
677\end{array}$ & 18.4 & $\begin{array}{l}48 \\
659\end{array}$ & 19.2 & $\begin{array}{l}13 \\
250\end{array}$ & 5.2 & $\begin{array}{l}253 \\
500\end{array}$ & 100.0 \\
\hline
\end{tabular}

a Percentage of column-specific total for each variable row, percentage of whole cohort in 'total' row

${ }^{b}$ Early and late readmissions are not mutually exclusive as they are captured across the whole time period 

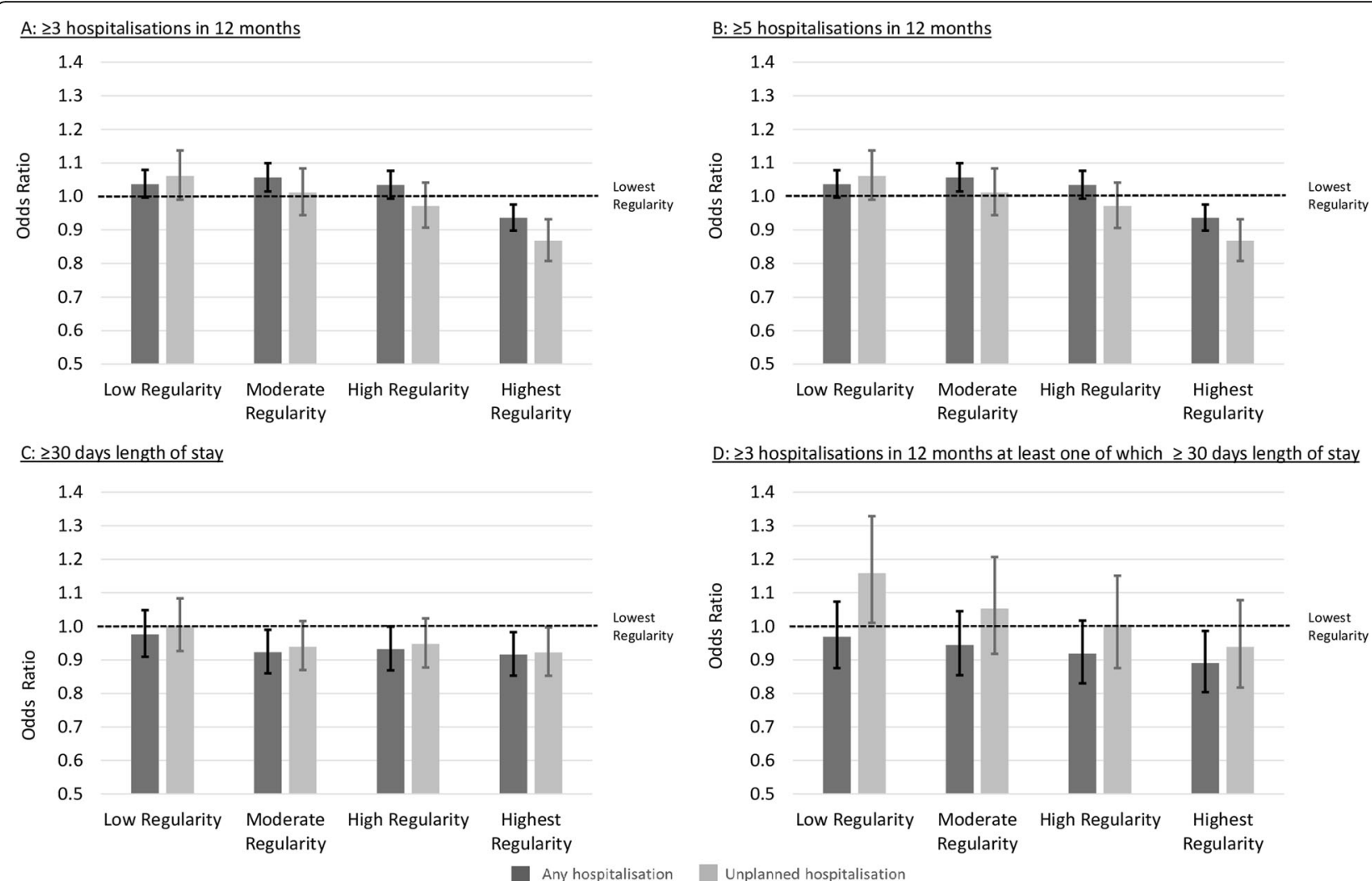

Fig. 1 Adjusted $^{a}$ odds ratios of (a) $\geq 3$ hospitalisations in hospital in 12 months; (b) $\geq 5$ hospitalisations in hospital in 12 months; (c) $\geq 30$ days length of stay and (d) $\geq 3$ stay in 12 months one of which $\geq 30$ days length of stay in the follow-up period (1 July 2009 to 31 December 2017) according to quintile of regularity during the exposure period (1 July 2005 to 30 June 2009). a Adjusted for: usual provider of care index, modified modified continuity index, frequency of general practitioner (GP) contact, number of chronic disease management contacts, number of mental health GP contacts, number of specialist physician contacts, sex, marital status, Indigenous status, living independently, alcohol use, born in Australia, physical activity level, time spent sitting, level of limitation, psychological distress, self-rated overall health, self-rated quality of life, social support, highest attained education level, household income, body mass index, smoking history, remoteness index, post (zip)-code based socioeconomic status, self-reported previously diagnosed medical conditions, comorbidity 1 and 5 years prior to the start of follow up, Rx-risk at 1 and 5 years prior to start of follow up, died during follow up and number of days out of hospital during the exposure period

days. However, when confined to unplanned hospitalisation, a reduction in odds was only observed for individuals in the highest quintile of regularity (OR 0.92, 95\% CI 0.85-1.00).

For the combined metric of $\geq 3$ hospitalisations of which $\geq 1$ had a LOS $\geq 30$ days, a reduction in odds was only observed for those with the highest regularity with respect to all hospitalisations (OR 0.89, 95\% CI 0.800.99 ), while being in the low regularity quintile was associated with a higher odds (OR 1.16, 95\% CI 1.01-1.33) for unplanned hospitalisation.

Only the highest regularity quintile was associated with a reduction in odds of readmission within 30 days for all (OR 0.92, 95\% CI 0.89-0.95) and unplanned (OR 0.83, 95\% CI 0.79-0.88) hospitalisations (Fig. 2, Additional file 4). The same pattern was observed for late readmissions with ORs of 0.92 (95\% CI $0.89-0.95)$ and 0.86 (95\% CI 0.80-0.92) for all and unplanned, respectively. In contrast, for early readmissions lower odds were seen for both high (OR 0.92, 95\% CI 0.86-0.99) and highest regularity (OR $0.86,95 \%$ CI $0.80-0.92)$ when restricted to unplanned hospitalisations. When all hospitalisations were considered, only the highest regularity was associated with lower early readmissions (OR 0.93, 95\% CI 0.89-0.97). Regardless of readmission type, for any hospitalisation, small increases in ORs above that plausibly due to chance were observed for those with low regularity.

The PAFs associated with a move to highest regularity were highest for unplanned hospitalisations (Fig. 3, Additional file 5). For all hospitalisations the PAF ranged from 0.05 ( $\geq 30$ LOS) to 0.08 ( $\geq 3$ hospitalisations with $\geq 30$ day LOS and for $\geq 5$ hospitalisations). By contrast, for unplanned hospitalisations the potential reduction in the number of individuals having an event ranged from 0.06 ( $\geq 3$ hospitalisations with $\geq 30$ LOS) to 0.13 ( $\geq 5$ hospitalisations). Additional file 5 shows the number of individuals who could have been prevented from having 


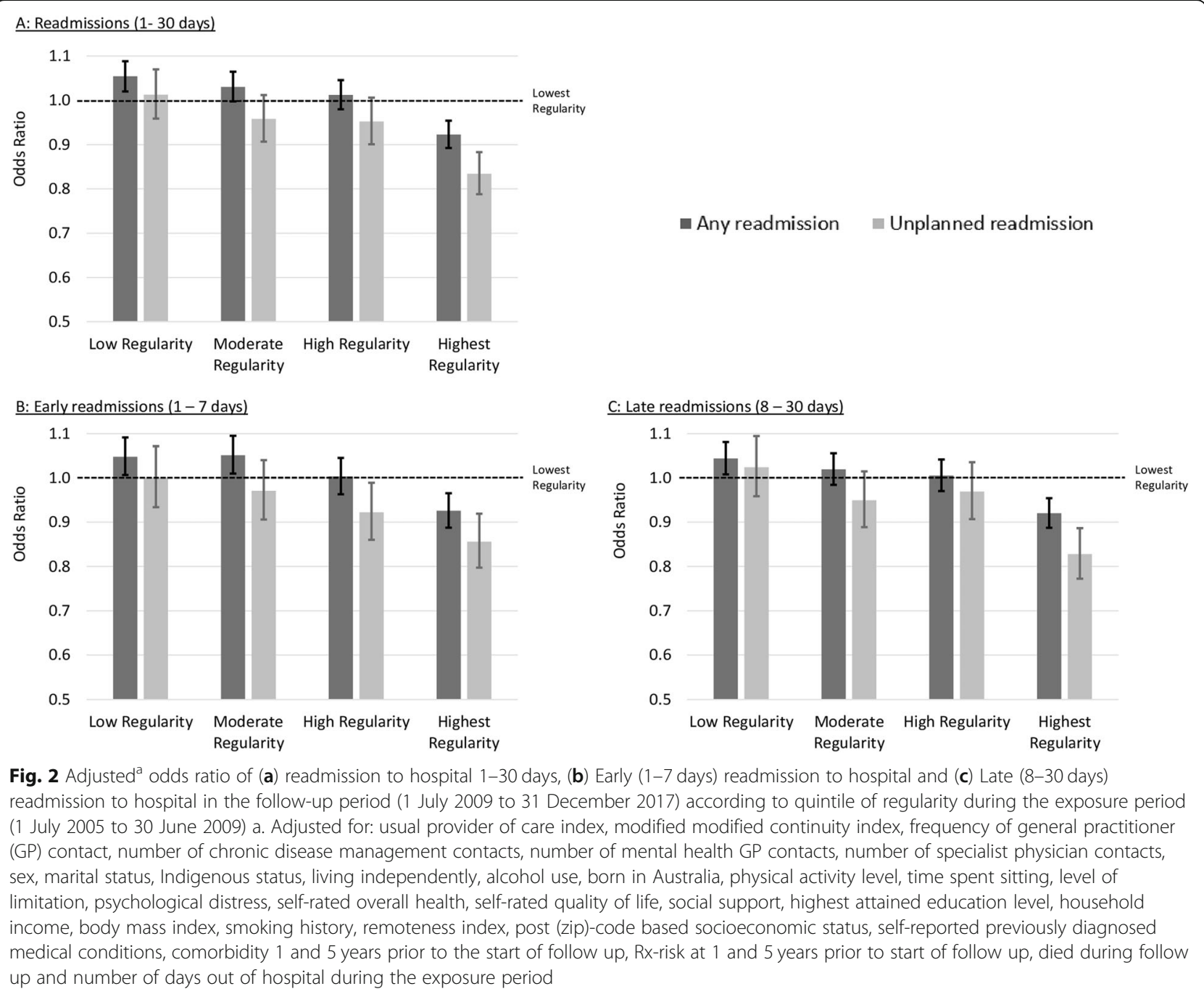

each event under study during the outcome ascertainment period, if they had been in the highest regularity quintile. This ranged from 269 ( $\geq 3$ unplanned hospitalisations with $\geq 30$ day LOS) to 2784 (30-day readmission) individuals.

\section{Discussion}

To our knowledge, this is the first study to examine the association between GP regularity and 'high use' hospitalisation. The results showed that compared with lowest regularity, highest regularity was associated with a reduction in the odds of 'high use'. With the exception of LOS $\geq 30$ days, the association was greater in magnitude for unplanned, relative to all hospitalisations. For LOS $\geq 30$ days for any hospitalisation, we observed a reduction in the odds of an individual having this outcome for all levels of regularity above low. Though we did not formally test for the presence of dose-response relationships, the results did not provide compelling evidence of a linear dose-response. For some analyses a significant effect was observed only at the highest level of regularity, for others effects were also observed at moderate levels and in one case coefficients for the moderate and high levels moved in opposite directions, compared to baseline."

\section{Comparison with previous findings}

Using U.S. data, Bazemore and colleagues [44] found higher continuity of provider was associated with lower hospital expenditure and hospital admission rates. Barker and colleagues [45] also found an inverse association between continuity of provider and hospitalisation for 22 'ambulatory care sensitive conditions' in the UK. Our study differs from these in focusing on GP regularity, adjusting for, rather than focusing on who is providing the care. In Australia, the 'cycle of care' for diabetes - payment for an annual suite of investigations - was associated with a $23 \%$ reduction in hospitalisation [46]. 


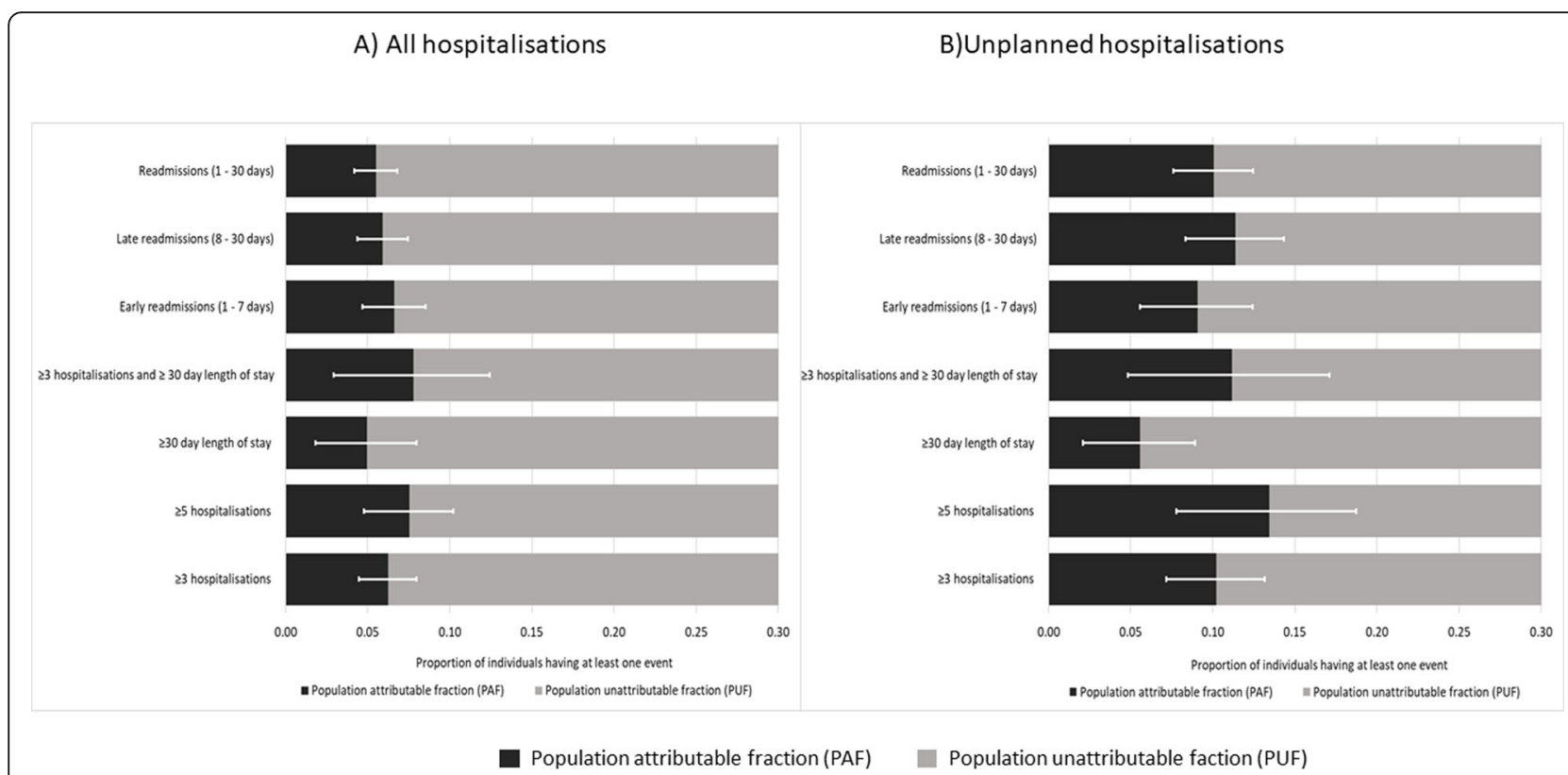

Fig. 3 Population attributable and unattributable fractions for general practitioner regularity, comparing the scenario with all individuals in the highest regularity quintile with the distribution as observed. Error bars are $95 \%$ confidence intervals

More regular primary care has been associated with reduced hospitalisation for people with diabetes [16], ischaemic heart disease $[15,47]$ and respiratory disease [12]. This study extends this finding to patterns of 'high use' hospitalisation. Our results are consistent with previous findings that primary care has the potential to reduce readmissions [21-24].

Unlike other studies evaluating rates or cost of hospitalisation $[12,16,20,44,47]$ we did not find a strong inverse association across increasing regularity levels. This may have been due to the outcomes we evaluated having a different relationship with regularity. The retrospective, cohort study design reported here increases the likelihood of a causal relationship in comparison to a crosssectional design because there is no risk of reverse causality [48]. However, a limitation of this design is that in health services research the 'real time' relationship between exposure and outcome is often of interest. The study design is therefore conservative, as the potential time lag between measurement of exposures and outcomes may diminish the ability to detect changes.

The plausibility of the association is another aspect in considering if the associations are causal. Authors of a recent systematic review concluded high cost users typically have multiple physical or mental health conditions [49]. While the results of our study are consistent with this view, in that more regular GP follow-up may prevent complications, this view is not unanimous. For instance, Lee and colleagues [50] conclude most people with frequent admission are not so-called "hot spotters" with poorly managed chronic conditions.

\section{Policy context and implications}

While ORs can estimate the strength of an association they do not provide information on the population event burden due to the underlying exposure. This can be estimated through the PAFs, though these values should be interpreted cautiously because there is a risk of unmeasured confounding affecting the observed associations. The PAFs indicate that for some outcomes, over $10 \%$ of individuals with an outcome was attributable to regularity, while for others the PAFs were much lower. Further, the results show even relatively modest PAFs can potentially result in large numbers of individuals with avoided 'high use' outcomes. The PAF for each outcome indicates factors other than regularity - even after extensive covariate adjustment - may be contributing factors. Using Australian private health insurance data, Khoo and colleagues [51] found many 'high use' hospitalisations were for unavoidable reasons (e.g. related to social considerations [52]). This is consistent with the discrepant results in this study between all and unplanned hospitalisations.

\section{Strengths and limitations}

This study linked self-report data from Australia's largest population-based cohort with administrative data [27]. Using a cohort who self-selected for a survey data reduces the generalisability of prevalence values, but not the internal validity with respect to associations identified [53]. A limitation of the regularity score is that it has no natural units and therefore is conventionally assessed using categories (e.g. quintiles) derived from 
the distribution found in the population of interest. Thus, the cut-off values for the quintiles are not standardised. This could affect the results in populations with a different distribution of regularity scores to that observed here. As with all observational studies there is potential for unobserved confounders to bias findings, although the self-report data allow for a wide range of characteristics to be controlled for. Finally, we evaluated outcomes using binary measures, so the association between regularity and the number of 'high use' events was not assessed.

\section{Conclusions}

This analysis showed that a very high GP regularity is associated with a decreased likelihood of individuals experiencing 'high use' hospitalisation, differentially for some outcomes between all and unplanned hospitalisations. This augments previous literature on continuity of provider, providing stimulus for further evaluation of regularity.

\section{Supplementary information}

Supplementary information accompanies this paper at https://doi.org/10. 1186/s12913-020-05718-0.

Additional file 1. Formulae used for continuity of GP contact metrics. Additional file 2. Categories of study covariates.

Additional file 3. Sociodemographic characteristics, by regularity quintile.

Additional file 4. Adjusted ${ }^{a}$ odds ratios (ORs) for general practitioner (GP) regularity and 'high use' outcomes.

Additional file 5. Potential number of individuals with avoided outcomes based on population attributable fraction estimates.

\section{Abbreviations \\ APDC: Admitted Patient Data Collection; DHS: Department of Human Services; GP: General practitioner; LOS: Length of stay; MACSS: Multipurpose Australian Comorbidity Scoring System; MBS: Medicare Benefits Schedule; MMCI: Modified Modified Continuity Index; NSW: New South Wales; OR: Adjusted odds ratio; PAF: Population attributable fraction; PBS: Pharmaceutical Benefits Scheme; PUF: Population unattributable fraction; RBDM: Register of Births Deaths and Marriages; RECORD: REporting of studies Conducted using Observational Routinely-collected health Data; UK: United Kingdom; UPC: Usual provider of care index; U.S: United States}

\section{Acknowledgments}

The 45 and Up Study is managed by the Sax Institute in collaboration with major partner Cancer Council NSW; and partners: the National Heart Foundation of Australia (NSW Division); NSW Ministry of Health; NSW Government Family \& Community Services - Ageing, Carers and the Disability Council NSW; and the Australian Red Cross Blood Service. We thank the many thousands of people participating in the 45 and Up Study. We also acknowledge the Commonwealth Department of Human Services for provision of the MBS and PBS data.

\section{Authors' contributions}

Due to data access restrictions placed on the 45 and Up study data only the approved analysts (RM and DY) had access to the data for analysis, while all remaining authors, external and internal, had full access to all statistical reports and tables. RM and DY can take responsibility for the integrity of the data, while all authors can take responsibility for the accuracy of the data analysis. CW and RM wrote the draft manuscript. All authors (RM, DY, DP and
CW) revised the manuscript for important intellectual content. The author(s) read and approved the final manuscript.

\section{Funding}

This study was part of a project funded by the Australian National Health and Medical Research Council (APP1078345). The study funders had no role in the study design, conduct, manuscript writing or decision to submit for publication.

\section{Availability of data and materials}

This research was completed using data collected through the 45 and Up Study (www.saxinstitute.org.au). The data that support the findings of this study are available from the Sax Institute, but restrictions apply to the availability of these data, which were used under license for the current study, and so are not publicly available. Information on the process for accessing these data can be found here: https://www.saxinstitute.org.au/ourwork/45-up-study/for-researchers/

\section{Ethics approval and consent to participate}

Ethical approval was obtained from Curtin University Human Research Ethics Committee (RD-42-14) and the NSW Population and Health Services Research Ethics Committee (HREC/17/CIPHS/37) and data custodian approval was granted for access to linked data.

Consent was given by participants in the 45 and Up Study for their information to be used in approved studies, and for follow-up and data linkage.

Consent for publication

Not applicable.

\section{Competing interests}

The authors declare that they have no competing interests.

\section{Author details}

${ }^{1}$ Health Economics and Data Analytics, School of Public Health, Faculty of Health Sciences, Curtin University, GPO Box U1987, Perth, Western Australia 6845, Australia. ${ }^{2}$ School of Population and Global Health, Faculty of Health and Medical Sciences, University of Western Australia, Perth, Western Australia, Australia. ${ }^{3}$ School of Medicine, College of Health \& Medicine, University of Tasmania, Hobart, Tasmania, Australia.

Received: 12 January 2020 Accepted: 7 September 2020

Published online: 06 October 2020

\section{References}

1. Duckett S. Expanding the breadth of Medicare: learning from Australia. Health Econ Policy Law. 2018;13(3-4):344-68.

2. Australian Government. Australia to 2050: future challenges. Canberra: Australian Government; 2010

3. Australian Government DoH. History of key MBS primary care initiatives 1999-2013. Canberra: Australian Government, Department of Health; 2014 [Available from: http://www.health.gov.au/internet/main/publishing.nsf/ Content/mbsprimarycare-History.

4. Joynt KE, Gawande AA, Orav EJ, Jha AK. Contribution of preventable acute care spending to total spending for high-cost Medicare patients. JAMA. 2013;309(24):2572-8.

5. Zook CJ, Moore FD. High-cost users of medical care. N Engl J Med. 1980; 302(18):996-1002.

6. Johansen $\mathrm{H}$, Nair $\mathrm{C}$, Bond J. Who goes to the hospital? An investigation of high users of hospital days. Health Rep. 1994;6(2):253-77.

7. Bayliss EA, Ellis JL, Shoup JA, Zeng C, McQuillan DB, Steiner JF. Effect of continuity of care on hospital utilization for seniors with multiple medical conditions in an integrated health care system. Ann Fam Med. 2015;13(2): $123-9$.

8. Menec VH, Sirski M, Attawar D, Katz A. Does continuity of care with a family physician reduce hospitalizations among older adults? J Health Serv Res Policy. 2006;11(4):196-201.

9. Jee $\mathrm{SH}$, Cabana MD. Indices for continuity of care: a systematic review of the literature. Med Care Res Rev. 2006;63(2):158-88. 
10. Breslau N, Haug MR. Service delivery structure and continuity of care: a case study of a pediatric practice in process of reorganization. J Health $\mathrm{Soc}$ Behav. 1976;17(4):339-52.

11. Magill MK, Senf J. A new method for measuring continuity of care in family practice residencies. J Fam Pract. 1987;24(2):165-8.

12. Einarsdóttir K, Preen D, Emery J, Kelman C, Holman C. Regular primary care lowers hospitalisation risk and mortality in seniors with chronic respiratory diseases. J Gen Intern Med. 2010;25(8):766-73.

13. Gibson D, Moorin R, Preen D, Emery J, Holman C. Effects of the Medicare Enahanced primary care program on primary care physician contact in the population of older Western Australians with chronic diseases. Aust Health Rev. 2011;35:334-40.

14. Gibson D, Moorin R, Preen D, Emery J, Holman C. Enhanced primary care improves GP service regularity in elderly patients without impacting on service delivery. Aust J Prim Health. 2012;18:295-303.

15. Australian Institute of Health and Welfare. Transition between hospital and community care for patients with coronary heart disease: New South Wales and Victoria, 2012-2015. Cat. no. CDK 9. Canberra: Australian Capital Territory, Australia: Australian Institute of Health and Welfare; 2018.

16. Youens D, Harris M, Robinson S, Preen DB, Moorin RE. Regularity of contact with GPs: measurement approaches to improve valid associations with hospitalization. Fam Pract. 2019;36(5):650-6.

17. Campbell SM, Kontopantelis E, Reeves D, Valderas JM, Gaehl E, Small N, et al. Changes in patient experiences of primary care during health service reforms in England between 2003 and 2007. Ann Fam Med. 2010;8(6):499506.

18. The King's Fund. Understanding pressures in general practice. London: UK The King's Fund; 2016.

19. Ladapo J, Chokshi DA. Continuity Of Care For Chronic Conditions: Threats, Opportunities, And Policy. Health Affairs blog; 2014.

20. Moorin $\mathrm{R}$, Youens $\mathrm{D}$, Preen DB, Wright CM. The association between continuity of provider-adjusted regularity of general practitioner contact and unplanned diabetes-related hospitalisation: a data linkage study in New South Wales, Australia using the 45 and up study cohort. BMJ Open. 2019;9: e027158.

21. Tang N, Maselli JH, Gonzales R. Variations in 30-day hospital readmission rates across primary care clinics within a tertiary referral center. J Hosp Med. 2014;9(11):688-94.

22. Avaldi VM, Lenzi J, Castaldini I, Urbinati S, Di Pasquale G, Morini M, et al. Hospital readmissions of patients with heart failure: the impact of hospital and primary care organizational factors in northern Italy. PLoS One. 2015; 10(5):e0127796.

23. White B, Carney PA, Flynn J, Marino M, Fields S. Reducing hospital readmissions through primary care practice transformation. J Fam Pract. 2014;63(2):67-73.

24. Jencks SF, Williams MV, Coleman EA. Rehospitalizations among patients in the Medicare fee-for-service program. N Engl J Med. 2009;360(14):1418-28.

25. Canadian Institute for Health Information. Defining high users in acute care: an examination of different approaches. Ottawa: Canadian Institute for Health Information; 2015

26. Benchimol El, Smeeth L, Guttmann A, Harron K, Moher D, Petersen I, et al. The REporting of studies conducted using observational routinely-collected health data (RECORD) statement. PLoS Med. 2015;12(10):e1001885.

27. Banks E, Redman S, Jorm L, Armstrong B, Bauman A, Beard J, et al. Cohort profile: the 45 and up study. Int J Epidemiol. 2008;37(5):941-7.

28. Lawrence G, Dinh I, Taylor L. The Centre for Health Record Linkage: a new resource for health services research and evaluation. Health Inf Manag. 2008;37(2):60-2.

29. Department of Health and Ageing. MBS online Canberra 2011 [Available from: http://www.health.gov.au/internet/mbsonline/publishing.nsf/Content/ Medicare-Benefits-Schedule-MBS-1.

30. Jung B, Cho KH, Lee DH, Kim S. The effects of continuity of care on hospital utilization in patients with knee osteoarthritis: analysis of Nationwide insurance data. BMC Health Serv Res. 2018;18(1):152.

31. Ionescu-Ittu R, McCusker J, Ciampi A, Vadeboncoeur AM, Roberge D, Larouche D, et al. Continuity of primary care and emergency department utilization among elderly people. CMAJ. 2007;177(11):1362-8.

32. Graham KL, Auerbach AD, Schnipper JL, Flanders SA, Kim CS, Robinson EJ, et al. Preventability of early versus late hospital readmissions in a National Cohort of general medicine patients. Ann Intern Med. 2018;168(11):766-74
33. Australian Institute of Health and Welfare. The Active Australia Survey: a guide and manual for implementation, analysis and reporting. Canberra: Australian Institute of Health and Welfare; 2003.

34. Kessler RC, Andrews G, Colpe LJ, Hiripi E, Mroczek DK, Normand SL, et al. Short screening scales to monitor population prevalences and trends in non-specific psychological distress. Psychol Med. 2002;32(6):959-76.

35. Rand Healthcare. 36-Item Short Form Survey Instrument (SF-36) Santa Monica, California, United States Rand Healthcare, 2019 [Available from: https://www.rand.org/health-care/surveys_tools/mos/36-item-short-form/ survey-instrument.html.

36. Koenig HG, Westlund RE, George LK, Hughes DC, Blazer DG, Hybels C. Abbreviating the Duke social support index for use in chronically ill elderly individuals. Psychosomatics. 1993;34(1):61-9.

37. Australian Bureau of Statistics. Census of population housing: Socioeconomic indexes for areas. Canberra: ABS; 2006. p. 2011.

38. Australian Bureau of Statistics. ABS Maps (Remoteness Structure). Canberra, Australia: Australian Bureau of Statistics; 2006, 2011 [8 June 2018]. Available from: http://stat.abs.gov.au/itt/r.jsp?ABSMaps.

39. Holman C, Preen D, Baynham N, Finn J, Semmens J. A multipurpose Australian comorbidity scoring system performed better than the Charlson index'. J Clin Epidemiol. 2005;58:1006-14.

40. Preen DB, Holman CD, Spilsbury K, Semmens JB, Brameld KJ. Length of comorbidity lookback period affected regression model performance of administrative health data. J Clin Epidemiol. 2006;59(9):940-6.

41. Pratt NL, Kerr M, Barratt JD, Kemp-Casey A, Kalisch Ellett LM, Ramsay E, et al. The validity of the Rx-risk comorbidity index using medicines mapped to the anatomical therapeutic chemical (ATC) classification system. BMJ Open. 2018:8(4):e021122

42. Statacorp. Stata statistical software: release 16. College Station, Texas, United States: 2019.

43. Newson RB. Attributable and unattributable risks and fractions and other scenario comparisons. Stata J. 2013;13:672-98.

44. Bazemore A, Petterson S, Peterson LE, Bruno R, Chung Y, Phillips RL Jr. Higher primary care physician continuity is associated with lower costs and hospitalizations. Ann Fam Med. 2018;16(6):492-7.

45. Barker I, Steventon A, Deeny SR. Association between continuity of care in general practice and hospital admissions for ambulatory care sensitive conditions: cross sectional study of routinely collected, person level data. BMJ. 2017;356:j84.

46. Comino EJ, Islam MF, Tran DT, Jorm L, Flack J, Jalaludin B, et al. Association of processes of primary care and hospitalisation for people with diabetes: a record linkage study. Diabetes Res Clin Pract. 2015;108(2):296-305.

47. Einarsdottir K, Preen DB, Holman CDJ, Emery J. Regular primary care plays a significant role in secondary prevention of ischemic heart disease in a Western Australian cohort. J Gen Intern Med. 2011;26(10):1092-7.

48. Rubin DB. For objective causal inference, design trumps analysis. Ann Appl Stat. 2008;2(3):808-40

49. Wammes JJG, van der Wees PJ, Tanke MAC, Westert GP, Jeurissen PPT. Systematic review of high-cost patients' characteristics and healthcare utilisation. BMJ Open. 2018;8(9):e023113.

50. Lee NS, Whitman N, Vakharia N, Taksler GB, Rothberg MB. High-cost patients: hot-spotters Don't explain the half of it. J Gen Intern Med. 2017; 32(1):28-34

51. Khoo J, Hasan H, Eagar K. Examining the high users of hospital resources: implications of a profile developed from Australian health insurance claims data. Aust Health Rev. 2018;42(5):600-6.

52. Stokes J, Riste L, Cheraghi-Sohi S. Targeting the 'right' patients for integrated care: stakeholder perspectives from a qualitative study. J Health Serv Res Policy. 2018;23(4):243-51.

53. Mealing NM, Banks E, Jorm LR, Steel DG, Clements MS, Rogers KD. Investigation of relative risk estimates from studies of the same population with contrasting response rates and designs. BMC Med Res Methodol. 2010; 10:26.

\section{Publisher's Note}

Springer Nature remains neutral with regard to jurisdictional claims in published maps and institutional affiliations. 